\title{
REPRESENTACIONES SOCIALES DE DUPLAS PSICOSOCIALES SOBRE LAS ACCIONES PROFESIONALES REALIZADAS EN ESTABLECIMIENTOS MUNICIPALIZADOS, CHILLÁN, CHILE
}

\section{Carmen Gloria Jarpa-Arriagada ${ }^{1}$, Constanza Escobar ${ }^{2}$, Laura Guiñez ${ }^{3}$, Karla Salazar ${ }^{4}$}

\section{RESUMEN}

El artículo analiza las representaciones sociales de duplas psicosociales de escuelas municipales y discute la definición y objetivo de este dispositivo de intervención, que ingresa a las escuelas chilenas como una más de las medidas de inclusión social. Afirmamos que la escuela, como espacio de desarrollo humano, debe hacerse cargo de las inequidades que impiden a los niños más vulnerables lograr su plena integración escolar. Sin embargo, la mera instalación de la dupla psicosocial en las escuelas no parece suficiente. A través de entrevistas grupales nos aproximamos a la representación de las duplas psicosociales para comprender a qué se dedican y cómo se integran en el espacio escolar. Los resultados indican que el proceso de inserción a las escuelas ha sido dificultoso y que depende en exceso de las peculiaridades y demandas específicas de cada escuela. Existe resistencia del profesorado a la actuación de las duplas, y se les solicita actividades de baja calificación. Los hallazgos indican una amplia variabilidad en las funciones y una carga excesiva de funciones administrativas. Entonces, la labor de inclusión educativa queda cooptada por una variedad de obligaciones que desvían la atención de la dupla sobre el propósito principal de trabajar en la desigualdad e inequidad educativa.

Conceptos clave: dupla psicosocial, inclusión, intervención psicosocial, convivencia escolar.

1 Universidad del Bío-Bío, Chillán, Chile. Contacto: cjarpa@ubiobio.cl

2 Universidad del Bío-Bío, Chillán, Chile. Contacto: constanza.escobar1501@alumnos. ubiobio.cl

3 Universidad del Bío-Bío, Chillán, Chile. Contacto: laura.guinez1501@alumnos.ubiobio.cl

4 Universidad del Bío-Bío, Chillán, Chile. Contacto: karla.salazar1501@alumnos.ubiobio.cl 


\title{
SOCIAL REPRESENTATIONS OF PSYCHOSOCIAL PAIRS ON PROFESSIONAL ACTIONS CARRIED OUT IN MUNICIPALIZED SCHOOLS, CHILLÁN, CHILE
}

\begin{abstract}
The article analyzes the social representations of psychosocial pairs of municipal schools and discusses the definition and objective of this intervention device, which enters Chilean schools as one of the measures of social inclusion. We affirm that the school, as a space for human development, must assume responsibility for the inequities that prevent the most vulnerable children from becoming fully integrated into school life. However, the mere installation of the psychosocial duo in schools does not seem sufficient. Through group interviews, we approach the representation of psychosocial pairs to understand their work and how they are integrated into the school space. The results indicate that the process of insertion into schools has been difficult and excessively depends on the peculiarities and specific demands of each school. Teachers demonstrate resistance to the pairs' actions, and ask them to carry out low-skill activities. The findings indicate a wide variability in functions and an excessive burden of administrative functions. Thus, the work of educational inclusion is co-opted by a variety of administrative obligations that divert the pair's attention from the main purpose of working on educational inequality and inequity.
\end{abstract}

Key concepts: psychosocial pair, inclusion, psychosocial intervention, school coexistence. 


\section{Introducción}

Las escuelas chilenas incorporaron el dispositivo de intervención denominado "dupla psicosocial" a partir de 2008, fecha en que se promulgó la Ley 20.248 de Subvención Escolar Preferencial, conocida como ley SEP. Es preciso indicar que esta incorporación no es explícita en la ley, pero permite a los sostenedores la contratación de profesionales que se integren en las escuelas a trabajar en convivencia escolar. De esta manera, la incorporación de la dupla psicosocial al sistema educativo chileno queda anclada, simbólicamente, al reconocimiento de la vulnerabilidad social existente en muchas de las escuelas (Gatica, 2016) y a la necesidad de otorgar atención especializada a niños, niñas y adolescentes (NNA) cuyos resultados académicos pueden verse afectados por sus condiciones socioeconómicas. En este escenario, los NNA de estas escuelas son calificados como prioritarios o preferentes para el Ministerio de Educación en Chile.

Ciertamente, la dimensión político-normativa ha tenido un impacto notable en la configuración de la nueva institucionalidad escolar en Chile. En efecto, algunas leyes y normativas recientes que han impactado en la nueva estructura de las escuelas son la Política Nacional de Educación Especial (2005), la Ley 20.248 de Subvención Escolar Preferencial (2008), Decreto 170 (2009), que fija las normas para otorgar subvención especial a estudiantes con Necesidades Educativas Especiales (NEE), la Política de convivencia escolar (2011), el Programa de Educación en Sexualidad, Afectividad y Género (2012), la Ley 20.609 de no discriminación (2012), el Proyecto de Ley de migración y extranjería (2013), y la Ley 20.845 sobre la Inclusión Escolar (2015). Este entramado de recientes normas ha contribuido a forjar un nuevo discurso centrado en ideas de "inclusión", "igualdad" y "perspectiva de derecho", pero, a su vez, revela una evidente lógica de nuevo management público (Infante et al., 2011; Infante $\&$ Matus, 2009; Matus \& Infante, 2011) que ha impactado fuertemente en las escuelas de Chile.

El reconocimiento de la vulnerabilidad social existente en las escuelas públicas de Chile desnuda, explícitamente, el desafío 
ineludible de atender eficazmente a los niños y jóvenes en riesgo social, y que aparecen como población preferente y prioritaria. En este sentido, Fusarelli y Lindle (2011) plantean la necesidad de políticas sociales sistemáticas, porque las escuelas ya no pueden garantizar por sí solas el bienestar de los estudiantes. De este modo, la creación de programas interdisciplinarios que amplían la mera oferta educativa de las escuelas hacia intervenciones de tipo psicosocial, parece ser ineludible y trascendente, más aún en un país como Chile, que lidera los rankings de desigualdad del planeta.

En este contexto, la dupla psicosocial en Chile se inserta en las escuelas a través de tres distintos programas: el Programa de Integración Escolar (PIE), la Subvención Escolar Preferencial (SEP) y el Fondo de Apoyo a la Educación Pública (FAEP), prestando atención a los/las niños/as, adolescentes y jóvenes más vulnerables del país. El objetivo de este artículo es ilustrar las representaciones sociales que las duplas psicosociales de siete establecimientos educacionales municipales de la ciudad de Chillán tienen respecto de las acciones profesionales desarrolladas en las escuelas. Los hallazgos permiten discutir la inserción de este dispositivo en el espacio escolar y la compleja interacción interna que vivencian en un sistema que se caracteriza por su clausura disciplinar (Arnold-Cathalifaud, 2003; Mascareño, 2006, 2018). La reflexión a partir de los resultados nos lleva a afirmar que la atención psicosocial de niños, niñas y adolescentes vulnerables en Chile requiere de una de-construcción de los dispositivos de control y normalización, para situar mecanimos como la dupla psicosocial en la dimensión de la convivencia escolar.

\section{La escuela como dispositivo de poder}

Históricamente, el control social se ha configurado como formador de procesos organizacionales y sociales que pretenden ordenar la conducta humana con el fin de establecer hábitos colectivos. Algunos investigadores identifican como instrumentos de control social a las creencias, costumbres, expectativas y prácticas sociales que modelan el comportamiento de los individuos (Agamben, 2011; Giraldo, 2006; Redón, 2011; Romero et al., 2002; Vercellino, 2016); en este sentido, el control social constituye un mecanismo que busca reglamentar y estandarizar las conductas humanas. 
Algunos estudios realizados en América Latina confirman que el sistema educativo moldea las conductas, acciones y pensares de los individuos, alineándose como estrategia de crecimiento de productividad y eficiencia en la sociedad (Bravo, 2012; Jiménez, 2003; Santiago, 2017; Vercellino, 2016); en otras palabras, la escuela establece reglas y normativas que la estructuran como un dispositivo de control alineado con la lógica mercadista y el nuevo management público en los procesos educativos (Corvalán et al., 2016; Fuller, 2019; Madrid, 2016; Neumann, 2018; Villalobos y Quaresma, 2015).

La escuela ejerce un poder importante en los individuos porque, mediante las enseñanzas y prácticas impartidas en los centros educativos, los sujetos se constituyen y se redefinen. Diversos autores indican que los establecimientos educativos generan prácticas decididas, más o menos sofisticadas, para que el saber se constituya en una forma de poder, lo que devela una estrategia de subordinación desde el conocimiento, ejerciendo influencia significativa en las perspectivas de los individuos, en la forma de interpretar y posicionarse como sujetos (Boccasius, 2008; Done $\&$ Murphy, 2018; Gimeno Sacristán, 2000; Peña et al., 2017; Terigi, 2006; Valle y Jiménez, 2017).

En este sentido, los establecimientos educacionales se establecen como instituciones imprescindibles en la vida de las personas, les permite crecer y desarrollarse a pesar de las restricciones y estándares sociales a los que los establecimientos se adscriben. Por esto, desde la etapa preescolar, la escuela cumple un papel interventor en los procesos de socialización de las personas.

\section{La escuela como dispositivo para normalizar}

La escuela, como dispositivo de control social, tiende a ubicar a los sujetos dentro de parámetros estandarizados, es decir, divide a los estudiantes y los sitúa en categorías: los que están "dentro de la norma" o normalidad, y los que se desmarcan o desvían de las medidas comunes y generales. Ciertamente, el sistema educativo es violento y discriminador en relación con las diferencias existentes en los individuos, puesto que persigue valores y prácticas universales que son selectas para el grupo dominante. En definitiva, los 
establecimientos educativos se articulan con el sistema, para tener la capacidad de controlar y mantener la reproducción de aspectos sociales que se han significado como diferentes, con la finalidad de normalizarlos en el medio escolar (Anzaldúa y Yurén, 2011; Apablaza, 2017b; Lozano, 2011; Redón, 2011).

La escuela tiende a normalizar las diferencias o lo denominado "distinto", "insano" o "fuera de norma", en virtud de que la institución permanezca siendo tradicional, rutinaria y autoritaria, con la finalidad de mantener el control más que cimentar los aprendizajes de los alumnos. Algunas investigaciones revisadas dan cuenta de cómo la escuela no solo normaliza las diferencias sociales, sino que además posee un carácter reproductor de éstas (Anzaldúa y Yurén, 2011; López et al., 2012). En efecto, los establecimientos educativos intervienen condicionados por la lógica de normalización de las conductas "desviadas" o diferentes, con el objetivo de mantener un orden en el contexto escolar.

\section{La intervención psicosocial en las escuelas}

Para Apablaza (2014, 2016, 2017a, 2017b), la escuela funciona como un dispositivo de gobierno en el que predomina la racionalidad instrumental heredada del positivismo, con las inmediatas consecuencias de modos de subjetivación que no logran hacerse cargo del nuevo régimen de poder centrado en la inclusión, la igualdad y la perspectiva de género, suscitando serias limitaciones para la aceptación de las diferencias. Apablaza (2017b) identifica tres nudos en las escuelas en lo que respecta a intervención psicosocial: (i) la política de convivencia escolar, específicamente el abordaje de la violencia; (ii) las necesidades educativas especiales y el cumplimiento de la "inclusión", y (iii) las brechas de género. En efecto, los resultados de sus investigaciones se inclinan a mostrar que, no obstante disponer de una abundante normativa pro inclusión, los problemas psicosociales en las escuelas se siguen trabajando con modelos psicodinámicos de intervención, lo que ella denomina tecnologías "Psi" (Apablaza 2017a). En términos simples, la validación de lo "Psi" en las escuelas normaliza que lo que se tiene que hacer es psiquiatrizar/psicologizar la intervención, bajo el supuesto de que 
lo que está "perturbado" es el individuo, esto es, que los hándicaps están en el sujeto. Niega, de esta manera, la fuerza o potencia de las interacciones, en una tensión que por siglos ha alimentado la relación antagónica entre lo psicodinámico y lo sistémico.

Ciertamente, si en Chile el ingreso de las duplas psicosociales a las escuelas se produjo en el marco de leyes que ponen como población objetivo a los/las niños/as con necesidades educativas especiales, a los que se encuentran entre los más vulnerables dentro de los vulnerables, a los que están excluidos y, para incluirlos, se requiere de una acción decidida del Estado, podría explicarse la natural instalación de una lógica de intervenciones "Psi". Bajo este paradigma, a los profesionales se les permite actuar desde un saber experto, sobrepatologizando a las personas y a las familias, y actuando desde un dispositivo metodológico que implica estudiar, analizar, pesquisar e intervenir, reproduciendo la lógica de "objetos de estudio" más que de "actores sociales". En definitiva, una actuación profesional de este tipo "incluye" a los sujetos en tanto son beneficiarios de un plan de acción, pero los "excluye" ya que los estigmatiza, patologiza y etiqueta en categorías como "pobre", "incompetente", "disruptivo", "disfuncional", "violento", "integrado", entre otras.

Otro antecedente para discutir la instalación de las duplas psicosociales en las escuelas chilenas es el grado de coherencia entre lo que plantea la "letra" de la ley (o leyes) y la implementación específica en cada contexto escolar. Los hallazgos internacionales muestran que, cuando de intervención psicosocial escolar se trata, los efectos de cualquier programa van a depender directamente de las características organizativas de la escuela, de su composición y de su relación con el entorno (Manning, 2017; McCarty-Caplan, 2018; Palosaari, 2018; Trach et al., 2018; Velki, 2018; Webb et al., 2017).

\section{Metodología}

\subsection{Enfoque epistemológico}

Las representaciones sociales se utilizan como enfoque epistemológico, toda vez que son comprendidas como la forma en que el discurso socialmente elaborado y compartido entre los individuos se 
transforma en conocimiento (Apablaza, 2014; Jarpa-Arriagada y Castañeda, 2018; Valencia, 2007). En consecuencia, las duplas psicosociales construyen una realidad socialmente compartida a través de sus valores, cultura, ideología y comunicación, definida en las interacciones que sostienen y en los contextos en que se desenvuelven. En definitiva, las representaciones sociales se construyen a partir de los procesos de interacción y comunicación social, y se materializan en prácticas sociales (Abric, 2001; Araya, 2002; Rodríguez, 2003); es decir, las conductas y comportamientos de los individuos emergen desde el conocimiento que adquieren en la convivencia con otros sujetos. Ciertamente, las representaciones sociales se traducen en acciones y labores que las duplas psicosociales ejecutan, a partir de la interpretación de los saberes, ideas y conocimientos obtenidos en la vida cotidiana (Arancibia et al., 2013; Armijo Cabrera, 2019; Rodríguez, 2007).

\subsection{Método}

El método corresponde a estudio de casos instrumental, de tipo colectivo (Stake, 2010). La elección del método obedeció a contactos previos hechos desde la Universidad del Bío-Bío con el Departamento de Administración de Educación Municipal (DAEM) de la comuna en la que se hizo el estudio. Estos contactos se hicieron por intermedio de un grupo de investigación denominado "Familia, Escuela y Sociedad" (FESOC) y, a partir de estos contactos, existía buena disposición de varias duplas psicosociales, que fue manifestada de manera inicial en una reunión de difusión y sensibilización del tema y luego refrendada con la firma del consentimiento informado por siete duplas psicosociales de la comuna de Chillán.

\subsection{Participantes}

El sujeto de estudio fue la dupla psicosocial, siendo siete (7) las duplas participantes de la investigación. Los criterios de inclusión fueron la voluntariedad, la trayectoria de mínimo seis meses trabajando en conjunto y que cumplieran su labor en escuelas municipales de la ciudad de Chillán. 
La tabla 1 contiene la información detallada al respecto. Los nombres de las escuelas y de las duplas son ficticios:

Tabla 1.

Escuelas y duplas psicosociales participantes en el estudio

\begin{tabular}{ll}
\hline Escuela & Dupla psicosocial \\
\hline Las Rosas & Trabajadora social: Emilia \\
& Psicóloga: Pía \\
& Tiempo de la dupla actual trabajando en conjunto: 3 años \\
\hline Las Camelias & Trabajadora social: Bárbara \\
& Psicóloga: Leonor \\
& Tiempo de la dupla actual trabajando en conjunto: 6 meses \\
\hline El Tulipán & Trabajadora social: Isidora \\
& Psicóloga: Ana \\
& Tiempo de la dupla actual trabajando en conjunto: 1 año \\
\hline Las Violetas & Trabajadora social: Julia \\
& Psicólogo: David \\
& Tiempo de la dupla actual trabajando en conjunto: 2 años \\
\hline El Clavel & Trabajadora social: Natalia \\
& Psicóloga: Mónica \\
& Tiempo de la dupla actual trabajando en conjunto: 5 años \\
\hline Las Hortensias & Trabajador social: Rodrigo \\
& Psicólogo: Damián \\
& Tiempo de la dupla actual trabajando en conjunto: 5 años \\
\hline El Girasol & Trabajadora social: Luciana \\
& Psicóloga: Carmen \\
& Tiempo de la dupla actual trabajando en conjunto: 7 años \\
\hline
\end{tabular}

\subsection{Técnica de producción de datos}

La técnica de producción de datos fue la entrevista semiestructurada grupal, requiriendo la presencia y participación de ambos profesionales miembros de la dupla psicosocial (trabajador/a social y psicólogo/a). Se optó por su desarrollo en modalidad grupal (Valles, $1997,2007)$ con el fin de conocer el discurso y las significaciones que la díada construía a partir de su acción profesional en el ámbito educativo.

\subsection{Técnica de análisis de datos}

El análisis del discurso fue la estrategia de interpretación de los datos, entendida como una actividad científica versátil y especialmente útil en estudios ligados a representaciones discursivas, que permite 
la categorización a priori y la posibilidad de integrar categorías emergentes durante todo el proceso, conformándose como una estrategia que requiere de constante revisión y mirada analítica ante elementos no pronosticados (Santander, 2011; Sayago, 2014). El procedimiento implicó grabación de la entrevista semiestructurada y posterior transcripción; luego se realizó una codificación selectiva del corpus textual, asociando colores distintivos a cada categoría a priori, dando lugar a la identificación de categorías emergentes; construcción de matriz por cada entrevista, en la cual se agrupan los relatos de acuerdo con las categorías correspondientes; construcción de matriz general, elaborada con la totalidad de los enunciados por cada entrevista; producción de resultados, interpretación y elaboración de conclusiones.

Las categorías a priori fueron:

a) Funciones profesionales de la dupla psicosocial:

- Definición del trabajo en dupla psicosocial.

- Roles desempeñados por las duplas psicosociales.

- Acciones profesionales ejecutadas por las duplas psicosociales.

b) Elementos subyacentes a la práctica de la dupla y sus funciones:

- Mandatos de la escuela.

- Mandato del programa del que dependen administrativamente las duplas psicosociales.

- Perfil de la población atendida.

- Definición de la intervención psicosocial.

- Lógica de las profesiones.

- Obstaculizadores del quehacer profesional de las duplas psicosociales.

c) Finalidad de la dupla psicosocial inserta en el contexto educativo.

\subsection{Criterios de calidad}

Los criterios de calidad fueron la credibilidad y la auditabilidad (Vasilachis, 2006). La credibilidad se logró mediante observaciones y conversaciones prolongadas con los participantes del estudio, y la revisión del corpus y confirmación de cada dupla psicosocial 
participante. Además, se mantuvo un compromiso con el trabajo de campo y esmero por la obtención de datos ricos. La auditabilidad supuso la revisión constante del proceso por parte de un investigador colaborador.

\subsection{Rigor ético de la investigación}

El rigor ético tuvo en cuenta la integridad de los sujetos de estudio, llevando a cabo un proceso voluntario, ratificado mediante consentimiento informado. Además, se aseguró la confidencialidad, ya que el nombre de cada integrante de la dupla fue modificado, así como el nombre de la escuela en la que prestaba servicios profesionales.

\section{Resultados}

El artículo profundiza en la categoría a priori "Funciones profesionales de la dupla psicosocial", con 177 fragmentos codificados, específicamente en la subcategoría "Acciones profesionales desarrolladas por las duplas psicosociales", con 124 fragmentos codificados. En esta subcategoría aparecen cuatro campos semánticos: "convivencia escolar"; "trabajo en red"; "atención de emergentes" y "acciones fuera del ámbito de sus competencias".

\subsection{Convivencia escolar}

Las duplas psicosociales afirman que las acciones profesionales desarrolladas por ellas al interior de las escuelas están relacionadas con el área de convivencia escolar. Al respecto, señalan que velar por la "sana convivencia" al interior de los establecimientos educacionales es una acción primordial.

... tenemos la función de llevar a cabo todo el plan de convivencia escolar o gran parte, el área de convivencia escolar es muy amplio porque trabajan todos los estamentos, desde la dirección hasta los auxiliares... (Damián, Escuela Las Hortensias")

...se hacen talleres, tratando de que los niños tengan una mejor convivencia... (Leonor, Escuelas Las Camelias) 
122 REPRESENTACIONES SOCIALES DE DUPLAS PSICOSOCIALES SOBRE LAS ACCIONES PROFESIONALES REALIZADAS EN ESTABLECIMIENTOS MUNICIPALIZADOS, CHILLÁN, CHILE - C. Jarpa-Arriagada, C. Escobar, Laura Guiñez, K. Salazar

\subsection{Trabajo en red}

Con la finalidad de obtener mejores resultados en las intervenciones llevadas a cabo con los alumnos y sus familias, las duplas psicosociales llevan a cabo un trabajo en red. Lo que queda demostrado en los siguientes enunciados:

...Con el Ministerio Público, denuncias a la PDI, que es como el mismo nexo, también derivaciones a la OPD, que demora quizás más en llegar a ese tema, y a programas de seguimiento y más especializados, enfocado como a todas las duplas, pero así como que trabajemos más dentro del sector es con la posta, y todo lo que hay, los profesionales que ahí están, nutricionista, enfermero, psicólogo... (David, Escuela Las Violetas)

...Las redes, principalmente de SENAME y las colaboradoras de SENAME, PPF, PIE, OPD, PRM, todo lo que es SENAME, [I2: Sí], las residencias, [I2: las residencias] y también con lo que es el área de salud, consultorios, en este caso los CESFAM, [I2: o el hospital], o el hospital cuando ya corresponde una atención que ya es más especializada, pero generalmente es más con el CESFAM... (Natalia, Escuela El Clavel)

\subsection{Atención de emergentes}

El relato de las duplas indica que, de acuerdo con las necesidades y/o problemas que surjan al interior de los establecimientos educacionales, ejecutan diversas actividades o acciones profesionales. De este modo, lo contingente, lo apremiante y lo emergente de la escuela adquiere una potencia constructiva de las acciones profesionales de las duplas, incluso por sobre las declaraciones normativas o reglamentarias.

...Está dentro de las funciones, pero igual es a criterio, no es que tu tengas que hacer cien visitas en el año o que tengan todos los niños que tener una visita, no. Dependiendo de cómo sea la problemática, de cómo se den las entrevistas, ahí uno ve si amerita una visita, si voy sola, si vamos en dupla, todo depende de cada caso... (Isidora, Escuela El Tulipán) 
...Por ejemplo, estamos con un alumno y nos llaman de dirección, o a mi colega la llaman de dirección o de orientación, o el subdirector con otro problema y nosotros tenemos que partir y dejar ese caso y tomar otro y así... tratar de solucionar los problemas también conductuales que ocurren espontáneamente... (Ana, Escuela El Tulipán)

\subsection{Acciones fuera de su ámbito profesional}

Las duplas psicosociales relatan que deben ejecutar una gran variedad de acciones, por demanda de la escuela o por necesidad del alumnado, aunque no tengan relación directa con su definición de funciones:

... Nos ha tocado animar actos, nos ha tocado animar diferentes cosas, organizar, de los viajes pedagógicos, podemos hacer los proyectos escritos de los viajes pedagógicos que, a lo mejor, nosotros no tenemos nada que ver con eso, pero igual nos hacemos cargo... (Carmen, Escuela El Girasol)

... Hacer suspensiones, que no nos corresponde porque hay inspectores para eso... (Mónica, Escuela El Clavel)

\section{Discusión de resultados}

Las acciones profesionales desarrolladas por la dupla psicosocial giran en torno a la atención de la vulnerabilidad de los estudiantes, cuestión que en sí misma no es negativa, pero que confirma a la escuela como un dispositivo de poder y normalización (Apablaza, 2017a; Villalobos y Quaresma, 2015). En este sentido, es posible advertir que tanto trabajador social como psicólogo podrían estar siendo cooptados por la preeminencia del modelo clínico de intervención y sus ideas de profesionalismo, de saber experto y de manejo especializado de un conjunto de técnicas que los convierten en eruditos. A partir de ese encuadre, realizan un "trabajo en red" fuertemente conectado con instituciones judiciales, ligadas al Servicio Nacional de Menores y a Salud. Entonces, sigue predominando una acción remedial, clínica, normalizadora, en la que niños prioritarios y preferentes son sobreintervenidos por una multiplicidad de instituciones que intentan, principalmente, lograr su adaptación a la institución escolar más que la atención integral de sus necesidades infantiles. 
El propósito de los programas estatales (PIE-SEP) es combatir la pobreza e inequidad al interior de los establecimientos educacionales en nuestro país, abordando las dificultades socioeconómicas y cognitivas del alumnado (Armijo Cabrera, 2019; Cornejo Espejo, 2019; Gatica, 2016; Inostroza-Barahona y Lohaus-Reyes, 2019; López et al., 2012; Raczynski et al., 2013). A propósito de lo expuesto, las duplas psicosociales reconocen enfoques determinados de acción, tales como la atención de estudiantes con mayores índices de vulnerabilidad desde Ley SEP y brindar apoyo a los estudiantes con necesidades educativas especiales desde programa PIE; sin embargo, las acciones profesionales desempeñadas a diario al interior de las escuelas, independiente del programa al que estén adscritas, son autorrefenciales para disminuir las brechas sociales existentes y resguardar que las dificultades cognitivas no sean un impedimento para que los alumnos permanezcan en los centros educativos. Nuevamente, entonces, aparecen campos semánticos ligados al control, la normalización y la adaptación de los niños al sistema escolar (Anzaldúa y Yurén, 2011; Cornejo Espejo, 2019).

El campo semántico de la convivencia escolar, como acción de la dupla psicosocial, se debilita ante una mirada pragmáticotecnológica de la misma y una dedicación principal a acciones ligadas a la conmemoración del "día de la convivencia escolar", a realizar talleres o acciones remediales de resolución de conflictos. La unidad de significado ligada a la expertiz (López et al., 2011; Ossa, 2011; Sotomayor, 2015; Castillo y Rodríguez, 2016) hace que la escuela reconozca a trabajadores sociales y psicólogos una formación profesional especializada, y sanciona este mandato hacia las duplas (Jarpa-Arriagada et al., 2019); sin embargo, el encargado formal del área es otro profesional y, por supuesto, el que se lleva el crédito. En efecto, hacerse cargo de la convivencia escolar es una connotación residual al interior de las escuelas; forma parte de las nuevas demandas ligadas a la ley de inclusión, pero aún no goza de popularidad en la escuela.

La convivencia escolar sigue siendo en la escuela un relato en el borde; la razón central de la existencia de las escuelas sigue siendo la enseñanza y los actores principales, por antonomasia, los profesores. 
En este sentido, declaramos la presencia de clausura disciplinar en la escuela, la cual, desde la posición sociopoiética como constructivismo duro, implica clausura operativa, determinismo y acoplamiento estructural y autoinformación (Arnold-Cathalifaud, 2003). Luego, la escuela, como sistema social, está configurada por comunicaciones operativas cerradas y autorrefenciales, que tienen como propósito su mantenimiento y estabilidad. De esta manera, la incorporación de un nuevo dispositivo, como la dupla psicosocial, es rápidamente incorporado a las comunicaciones operativas y se utiliza como un mecanismo más de la permanencia y de la estabilidad de la escuela. Aunque las duplas psicosociales relatan ciertas resistencias iniciales, la retroalimentación y los enlazamientos recursivos de la comunicación (Arnold-Cathalifaud, 2003) desarrollados por la escuela terminan por conseguir el acoplamiento estructural y la permanente autorreferencia de la institución escolar.

Esta lectura hace sinergia con la noción residual de la convivencia, toda vez que aparece como un suplemento o añadidura (in-deseada) a la función principal de la escuela, que es la enseñanza. En este sentido, la llegada de las duplas a las escuelas ofrece una oportunidad para descargar este requerimiento en profesionales que disponen de una matriz cognitiva más robusta en la temática, pero que, paradojalmente, disponen de un contrato muy precario. La condición de ser personal a honorarios puede influir en la aceptación de los requerimientos adicionales sin un cuestionamiento explícito, atendida la fragilidad de su vínculo laboral. En suma, el sistema escuela opera autopoieticamente y logra introducir todos los dispositivos nuevos en la misma lógica autorreferencial.

Mantener la estabilidad del sistema escolar también se nutre de una extensa y agobiante dedicación a actividades emergentes y de acciones que están fuera (o deberían estarlo) del campo de acción de las duplas psicosociales. El tiempo dedicado a estas cuestiones forma parte de los mecanismos para concentrar a las duplas psicosociales en un ámbito administrativo y asistencial, desmontando el mandato inicial de hacerse cargo de la desigualdad e inequidad al interior del sistema educativo. Por consiguiente, el trabajo de las duplas psicosociales está tensionado por el contexto de vulnerabilidad en 
el que trabajan, sus precarias condiciones contractuales y las asiduas demandas con sentido de apremio que desestabilizan la agenda de largo plazo (Jarpa-Arriagada et al., 2019). Asimismo, las resistencias de parte de los docentes hacia su rol, su intrusión en lo pedagógico y la (in)definición de su mandato global, hace que queden relegados a los requerimientos de cada Dirección de establecimiento y al marco que establezcan las Direcciones de Administración de Educación Municipal. Esta sensación de (in)definición alimenta representaciones sociales de inestabilidad, de precariedad y de obligación de atender todo lo emergente, eclipsando el propósito mayor de disminuir la desigualdad.

\section{Consideraciones finales}

La convergencia de dos disciplinas (Trabajo Social y Psicología) que históricamente han estado vinculadas y su incorporación a la escuela bajo el dispositivo "dupla psicosocial", es una realidad emergente y de crecimiento exponencial en el contexto educativo chileno. Esta díada, compuesta por profesionales de las ciencias sociales, posee un origen difuso y escasamente sistematizado, puesto que su inserción se impulsa principalmente por la Ley de Subvención Escolar Preferencial y el Programa de Integración Escolar, cuerpos legales que han carecido de un adecuado seguimiento y evaluación (López, Carrasco, Morales y Ayala, 2011; Sotomayor, 2015; Castillo y Rodríguez, 2016; Gatica, 2016; Sandoval y Lamas, 2017; Urbina, Basualto, Durán y Miranda, 2017). Por lo tanto, la discontinuidad de procesos evaluativos respecto de las actividades profesionales realizadas por las duplas psicosociales insertas en educación, ha provocado que estas carezcan de lineamientos específicos de acción. Si bien se reconoce que el enfoque radica en elevar el nivel de bienestar educativo de manera integral, no existe una definición específica sobre el concepto "trabajo psicosocial en contextos educativos". A pesar de aquello, el desarrollo de este dispositivo continúa en proceso.

Los programas de intervención psicosocial y su eficacia están fuertemente influidos por la organización de la escuela como sistema autopoiético (Mascareño, 2006, 2018). Por tanto, las funciones de las duplas psicosociales van a estar sujetas a las demandas de cada 
escuela, y estas pueden mostrar una amplia variabilidad, pero siguen estando en el dominio de lo emergente y apremiante. En este sentido, se observa aún un predominio de lo que denominamos "clausura disciplinar pedagógica", entendida como el proceso dirigido a mantener la homeostasis del sistema, impidiendo o dificultando el ingreso de todo aquello que se represente como ajeno o exterior a la función principal de la escuela: la enseñanza. En consecuencia, la instalación de las duplas psicosociales en las escuelas ha reportado resistencias que pueden ser sofisticadas, pero que van obstaculizando las posibilidades de abrir espacios cada vez más intersticiales entre la enseñanza y el abordaje psicosocial. Entonces, un nuevo llamado de atención al sistema escolar es que pueden sobrevenir nuevas crisis, como resultado de las condiciones estructurales que son prescriptivas de excesiva autonomía, autoorganización y autopoiesis (Mascareño, 2018). En este sentido, Mascareño, indica que "la autopoiesis de la comunicación que sostiene el sistema es, a la vez, un mecanismo que puede conducirlo a su colapso" (2018, p. 131).

Sostenemos que la comprensión del fenómeno escolar debería dar paso a una mirada más compleja y total, capaz de mirar lo que hacen los profesores en su espacio de enseñanza, lo que pasa en las interacciones cotidianas y su expresión en la convivencia escolar, las relaciones de las escuelas con las madres, padres y apoderados, la intervención en red, la gestión de recursos humanos, en suma, la mirada pedagógica en su amplio sentido implicaría mirar a cada ser humano (niño/a, profesor, directivo, asistente de la educación, personal administrativo, padre, madre, apoderado/a) en su dimensión personal y colectiva. Por tanto, la mera declaración de normativas y leyes, situadas en un espacio macrosistémico, no necesariamente tiene la fuerza ortogonal suficiente para producir cambios estructurales en las escuelas ni en sus equipos pedagógicos o directivos. Muchas veces las nuevas leyes son leídas como intrusivas, si lo que proponen demanda cambios sustanciales a aquello que veníamos haciendo de la misma forma por un largo tiempo. La instalación de un sentido inclusivo y de un proyecto formativo humanizante requiere de una comprensión con una nueva matriz cognitiva: los logros educativos no están divorciados de la calidad de la convivencia escolar. En suma, la convivencia escolar debería ser la prioridad. 
La inexistencia de lineamientos transversales y estables que se configuren como orientadores de la intervención psicosocial, provoca que el quehacer profesional de la díada se reduzca a acciones reactivas ante necesidades emergentes en el contexto escolar, ejecutando su labor desde la intuición profesional en lugar de regirse bajo parámetros propios de la dupla psicosocial. Por ende, esta investigación logra develar aspectos trascendentales del ejercicio laboral de trabajadores sociales y psicólogos en tanto dupla de profesionales inserta en el ámbito educativo.

Afirmamos que el desafío, entonces, es estar disponibles a una construcción armónica de funciones que atienda a una visión global del fenómeno escolar. Sin duda, la férrea defensa de los territorios disciplinarios se vuelve cada vez una opción menos lúcida; al contrario, la demanda es avanzar hacia la interdisciplina, hacia el intersticio. Si se trata de duplas psicosociales, la interpelación está cada vez más inclinada a la construcción de un dispositivo de intervención que de cuenta de la complejidad de la relación niño/aescuela-familia-comunidad, incorporando una visión ortogonal de la actuación profesional con firme foco en la transformación social. La desigualdad y la exclusión, como fenómenos complejos, requieren intervenciones complejas. Derribar las barreras de la clausura disciplinar resulta urgente.

Al finalizar y a modo de propuesta, Stone (2015) postula que las principales directrices sobre las que se debería construirse una intervención multiprofesional en la escuela son: (i) vínculos entre el hogar, la escuela y la comunidad, (ii) directrices éticas y políticas educativas, (iii) defensa y derechos de educación, y (iv) toma de decisiones basada en datos. Es posible concluir que nuestro país está en una etapa muy inicial respecto de este horizonte, pero existen señales que podrían estar en consonancia con las experiencias ya consolidadas en otros países. En este sentido, la dupla psicosocial podría convertirse en un dispositivo que se hiciera cargo de la convivencia escolar como dominio complejo de las relaciones vitales al interior de la escuela, en el entendido que la creación de un ser humano supone aprendizajes de calidad. La calidad de la educación debería llevarnos a redefinir las estrategias de intervención psicosocial 
y resituarlas en el espacio de la construcción de alteridad, respeto y humanidad.

\section{Referencias}

Abric, J.-C. (2001). Prácticas sociales y representaciones. México: Coyoacán.

Agamben, G. (2011). ¿Qué es un dispositivo? Sociológica, 73, 249-264.

Anzaldúa, J. y Yurén, T. (2011). La diversidad en la escuela. Prácticas de normalización y estrategias identitarias en el caso de estudiantes gay de nivel medio superior. Perfiles Educativos, 33(133), 88-113.

Apablaza, M. (2014). Representaciones sociales de profesores respecto de la diversidad escolar en relación a los contextos de desempeño profesional, prácticas y formación inicial. Estudios Pedagógicos, XI(1), $7-24$.

Apablaza, M. (2017a, agosto 9). Producción de las diferencias en la escuela: Análisis de la política de inclusión educativa chilena. Cuarto Congreso Interdisciplinario de Investigación en Educación, Santiago de Chile.

Apablaza, M. (2017b). Prácticas 'Psi' en el espacio escolar: nuevas formas de subjetivación de las diferencias. Psicoperspectivas. Individuo y Sociedad, 16(3). DOI: https://doi.org/10.5027/psicoperspectivas-Voll6-Issue3fulltext-1063

Apablaza, M. (2016). Discursos de diferencia en educación: análisis de la política de diversidad/inclusión Chile. Investigação Qualitativa em Educação, 1, 336-345.

Arancibia, S., Rodríguez, G., Fritis, R., Tenorio, N. y Poblete, H. (2013). Representaciones sociales en torno a equidad, acceso y adaptación en educación universitaria. Psicoperspectivas, 12(1), 116-138.

Araya, S. (2002). Las representaciones sociales: ejes teóricos para su discusión. Cuaderno de Ciencias Sociales, Facultad Latinoamericana de Ciencias Sociales (FLACSO), 127.

Armijo Cabrera, M. (2019). Micro-política de una escuela marginalizada: entre inclusión y estandarización. Universitas, 30, 191-209. DOI: https://doi. org/10.17163/uni.n30.2019.09

Arnold-Cathalifaud, M. (2003). Fundamentos del constructivismo sociopoiético. Cinta de Moebio, 162-173.

Boccasius, A. (2008). Prácticas disciplinares en la Escuela. REXE. Revista de Estudios y Experiencia en Educación, 13, 95-102. 
130 REPRESENTACIONES SOCIALES DE DUPLAS PSICOSOCIALES SOBRE LAS ACCIONES PROFESIONALES REALIZADAS EN ESTABLECIMIENTOS MUNICIPALIZADOS, CHILLÁN, CHILE - C. Jarpa-Arriagada, C. Escobar, Laura Guiñez, K. Salazar

Bravo, P. (2012). Los muros de la sociedad de control: Por una emancipación del saber desde los saberes / The walls of the control society: for an emancipation of the wisdoms from the wisdoms. Sophia, 1(13), 154. DOI: https://doi.org/10.17163/soph.n13.2012.06

Castillo, V. y Rodríguez, C. (2016). Los aportes de los asistentes de la educación: la escuela como campo de intervención para el Trabajo Social. Cuaderno de Trabajo Social, 8, 62-84.

Cornejo Espejo, J. (2019). Nuevos excluidos en el sistema educacional chileno: problemas y desafíos. Páginas de Educación, 12(1), 28. DOI: https://doi.org/10.22235/pe.v12il.1766

Corvalán, J., Carrasco, A. y García-Huidobro, J. (Eds.). (2016). Mercado Escolar y oportunidad educacional: libertad, diversidad y desigualdad. Santiago de Chile: Ediciones Universidad Católica de Chile.

Done, E. J. \& Murphy, M. (2018). The responsibilisation of teachers: A neoliberal solution to the problem of inclusion. Discourse: Studies in the Cultural Politics of Education, 39(1), 142-155. DOI: https://doi.org $/ 10.1080 / 01596306.2016 .1243517$

Fuller, K. (2019). "That would be my red line": An analysis of headteachers' resistance of neoliberal education reforms. Educational Review, 71(1), 31-50. DOI: https://doi.org/10.1080/00131911.2019.1522042

Fusarelli, B. C. \& Lindle, J. C. (2011). The Politics, Problems, and Potential Promise of School-Linked Social Services: Insights and New Directions from the Work of William Lowe Boyd. Peabody Journal of Education, 86(4), 402-415. DOI: https://doi.org/10.1080/0161956X.2011.597270

Gatica, F. (2016). Las intervenciones psicosociales en establecimientos educacionales municipales vulnerables bajo el marco de la Ley SEP: diseño, implementación y logros desde la perspectiva de actores claves. Revista Estudios de Políticas Públicas, 3(0). DOI: https://doi. org/10.5354/0719-6296.2016.41830

Gimeno Sacristán, J. (2000). La educación obligatoria: su sentido educativo y social. Madrid: Ediciones Morata.

Giraldo, R. (2006). Poder y resistencia en Michel Foucault. Tabula Rasa, 4 , 103-122.

Infante, M. \& Matus, C. (2009). Policies and practices of diversity: Reimagining possibilities for new discourses. Disability \& Society, 24(4), 437-445. DOI: https://doi.org/10.1080/09687590902879049

Infante, M., Matus, C. y Vizcarra, R. (2011). Razonando sobre la idea de diferencia en las políticas educativas chilenas. UNIVERSUM, 2(26), 143-163. 
Inostroza-Barahona, C. y Lohaus-Reyes, M. F. (2019). Inclusión y diversidad: nuevos desafíos para la política curricular chilena. Reflexiones desde la teoría curricular y la justicia social. Revista Internacional de Educación para la Justicia Social, 8(1), 151. DOI: https://doi.org/10.15366/ riejs2019.8.1.009

Jarpa-Arriagada, C. G., Escobar, C., Guiñez, L. y Salazar, K. (2019). Somos el Gary Medel de la selección: representaciones sociales de duplas psicosociales sobre sus finalidades en el contexto educativo municipal, Chile. Rumbos TS. Un espacio crítico para la reflexión en Ciencias Sociales, 20, 149-173.

Jarpa-Arriagada, C. G. J. y Castañeda, M. T. (2018). Representaciones sociales frente al proyecto de carrera profesional docente en Chile: Análisis estructural del discurso. Revista Brasileira de Educação, 23(0). DOI: https://doi.org/10.1590/s1413-24782018230075

Jiménez, L. (2003). La reestructuración de la escuela y las nuevas pautas de regulación del trabajo docente. Revista Mexicana de Investigación Educativa, 8(19), 603-630.

López, V., Carrasco, C., Morales, M. y Ayala, Á. (2011). El encapsulamiento de los psicólogos escolares y profesionales de apoyo psicosocial en la escuela. Revista Internacional Magisterio, 53.

López, V., Julio, C. y Pérez, M. V. (2012). Barreras Culturales para la inclusión: políticas y prácticas de integración en Chile. Revista de Educación, 363, 256-281. DOI: https://doi.org/10.4438/1988-592X-RE-2012-363-180

Lozano, J. (2011). La disciplina en la escuela secundaria: significados de alumnos en riesgo de exclusión. Actualidades Investigativas en Educación, 7(3). DOI: https://doi.org/10.15517/aie.v7i3.9291

Madrid, S. (2016). Diversidad sin diversidad: los colegios particulares pagados de élite y la formación de la clase dominante en una sociedad de mercado. En CEPPE (Ed.), Mercado Escolar y Oportunidad Educacional: Libertad, Diversidad y Desigualdad (pp. 269-299). Santiago de Chile: Ediciones Universidad Católica de Chile.

Manning, R. F. (2017). Place-Consciousness and Bronfenbrenner's Ecological Systems Model: A Discussion of Recurring Issues that Undermine the Teaching of Indigenous Histories in New Zealand and Australian Schools. The Australian Journal of Indigenous Education, 46(02), 148159. DOI: https://doi.org/10.1017/jie.2016.31

Mascareño, A. (2006). Die Alt-Und Jungluhmannianer. La autopoiesis de la comunicación acerca de la comunicación sistémica. En Observando sistemas. Nuevas apropiaciones y usos de la teoría de Niklas Luhmann (pp. 365-390). Santiago de Chile: RIL Editores, Fundación Soles. 
132 REPRESENTACIONES SOCIALES DE DUPLAS PSICOSOCIALES SOBRE LAS ACCIONES PROFESIONALES REALIZADAS EN ESTABLECIMIENTOS MUNICIPALIZADOS, CHILLÁN, CHILE - C. Jarpa-Arriagada, C. Escobar, Laura Guiñez, K. Salazar

Mascareño, A. (2018). De la crisis a las transiciones críticas en sistemas complejos: Hacia una actualización de la teoría de sistemas sociales. Theorein. Revista de Ciencias Sociales, 3(1), 109-143.

Matus, C. \& Infante, M. (2011). Undoing diversity: Knowledge and neoliberal discourses in colleges of education. Discourse: Studies in the Cultural Politics of Education, 32(3), 293-307. DOI: https://doi.org/10.1080/0 1596306.2011 .573248

McCarty-Caplan, D. (2018). LGBT-Competence in Social Work Education: The Relationship of School Contexts to Student Sexual Minority Competence. Journal of Homosexuality, 65(1), 19-41. DOI: https://doi. org/10.1080/00918369.2017.1310547

Neumann, J. W. (2018). How power really works in schools. Phi Delta Kappan, 99(8), 30-35. DOI: https://doi.org/10.1177/0031721718775675

Ossa, C. (2011). El rol del psicólogo educacional: la transición desde el paradigma de la simplicidad al paradigma de la complejidad. Revista Pequén, 1, 72-82.

Palosaari, E. (2018). Society, individual, family, and school factors contributing to child mental health in war: The ecological-theory perspective. Child Abuse \& Neglect, 84, 205-216. DOI: https://doi.org/10.1016/j. chiabu.2018.07.033

Peña, P., Sánchez, J., Ramírez, J. y Menjura, M. I. (2017). La convivencia en la escuela. Entre el deber ser y la realidad. Revista Latinoamericana de Estudios Educativos, 13(1), 129-152.

Raczynski, D., Muñoz, G., Weinstein, J. y Pascual, J. (2013). Subvención Escolar Preferencial (SEP) en Chile: Un intento por equilibrar la macro y micropolítica escolar. Revista Iberoamericana sobre Calidad, Eficacia y Cambio en Educación, 11(2), 164-193.

Redón, S. (2011). Escuela e identidad: Un desafío docente para la cohesión social. Polis (Santiago), 10(30), 447-476. DOI: https://doi.org/10.4067/ S0718-65682011000300021

Rodríguez, Ó. (2003). Las representaciones sociales: entretejidos de la razón y la cultura. Relaciones. Estudios de Historia y Sociedad, XXIV(93), 53-80.

Rodríguez, T. (2007). Sobre el estudio cualitativo de la estructura de las representaciones sociales. En Representaciones sociales. Teoría e investigación (p. 330). México: Universidad de Guadalajara.

Romero, A., Rujano, R. y Del Nogal, J. (2002). Control social: nuevas realidades, nuevos enfoques. Espacio Abierto, 11(4), 665-680. 
Sandoval, E. y Lamas, M. (2017). Impacto de la Ley SEP en las escuelas: Una mirada crítica y local en torno al rol de los psicólogos de la educación. Paideia, Revista de Educación, (61),57-81. ISSN: 0716-4815

Santander, P. (2011). Por qué y cómo hacer análisis de discurso. Cinta de Moebio, 41, 207-224.

Santiago, A. (2017). La sociedad de control: una mirada a la educación del siglo XXI desde Foucault. Revista de Filosofía, 73, 317-336. DOI: https://doi.org/10.4067/S0718-43602017000100317

Sayago, S. (2014). El análisis del discurso como técnica de investigación cualitativa y cuantitativa en las ciencias sociales. Cinta de Moebio, 49, 1-10. DOI: https://doi.org/10.4067/S0717-554X2014000100001

Sotomayor, M. (2015). Visibilizando el Trabajo Social Escolar: experiencias en la Región Metropolitana de Santiago, Chile. Revista Electrónica de Trabajo Social, Universidad de Concepción, 12, 43-56.

Stake, R. E. (2010). Investigación con estudio de casos (Cuarta). Madrid: Ediciones Morata.

Stone, S. (2015). School Social Work in the United States: Current Evidence and Future Directions. Arbor, 191(771), a201. DOI: https://doi. org/10.3989/arbor.2015.771n1003

Terigi, F. (2006). Diez miradas sobre la escuela primaria. Buenos Aires: Siglo Veintiuno Editores, Fundación OSDE.

Trach, J., Lee, M. \& Hymel, S. (2018). A Social-Ecological Approach to Addressing Emotional and Behavioral Problems in Schools: Focusing on Group Processes and Social Dynamics. Journal of Emotional and Behavioral Disorders, 26(1), 11-20. DOI: https://doi. org/10.1177/1063426617742346

Urbina, C., Basualto, P., Durán, C., y Miranda, P. (2017). Prácticas de co-docencia: El caso de una dupla en el marco del Programa de Integración Escolar en Chile. Estudios Pedagógicos (Valdivia), 43(2), 355-374. DOI: https://doi.org/10.4067/S0718-07052017000200019

Valencia, S. (2007). Elementos de la construcción, circulación y aplicación de las representaciones sociales. En Representaciones sociales. Teoría e investigación (p. 330). México: Universidad de Guadalajara.

Valle, A. M. y Jiménez, M. A. (2017). Escuela como fábrica de sujetos. Génesis de la modernidad escolar en México. ETD - Educação Temática Digital, 19(4), 642. DOI: https://doi.org/10.20396/etd.v19i4.8648659

Valles, M. (1997). Técnicas cualitativas de investigación social: Reflexión metodológica y práctica profesional. Madrid: Síntesis. 
134 REPRESENTACIONES SOCIALES DE DUPLAS PSICOSOCIALES SOBRE LAS ACCIONES PROFESIONALES REALIZADAS EN ESTABLECIMIENTOS MUNICIPALIZADOS, CHILLÁN, CHILE - C. Jarpa-Arriagada, C. Escobar, Laura Guiñez, K. Salazar

Valles, M. (2007). Entrevistas Cualitativas (Vol. 32). Centro de Investigaciones Sociológicas.

Vasilachis, I. (Ed.). (2006). Estrategias de investigación cualitativa (1 $1^{\mathrm{a}} \mathrm{ed}$ ). Barcelona: Gedisa Ed.

Velki, T. (2018). Verifying the ecological model of peer aggression on Croatian students: VELKI. Psychology in the Schools, 55(10), 1302-1320. DOI: https://doi.org/10.1002/pits.22178

Vercellino, S. (2016). Ampliación del tiempo y dispositivo escolar: oportunidades y resistências. Educação \& Realidade, 41(4), 1005-1025. DOI: https://doi.org/10.1590/2175-623661082

Villalobos, C. y Quaresma, M. L. (2015). Sistema escolar chileno: Características y consecuencias de un modelo orientado al mercado. Convergencia, 69, 63-84.

Webb, A., Canales, A. \& Becerra, R. (2017). Denying systemic inequality in segregated Chilean schools: Race-neutral discourses among administrative and teaching staff. Race Ethnicity and Education, 1-19. DOI: https://doi.org/10.1080/13613324.2017.1417254

Recibido: 05/07/2019

Aceptado:19/05/2020 